\title{
Absorption enhancement due to scattering by dipoles into silicon waveguides
}

\author{
K. R. Catchpole ${ }^{\text {a) }}$ and S. Pillai \\ School of Photovoltaic and Renewable Energy Engineering, University of New South Wales, \\ Sydney, New South Wales 2052, Australia
}

(Received 5 February 2006; accepted 15 May 2006; published online 22 August 2006)

\begin{abstract}
We develop an optical model for absorption enhancement and diffuse reflectance by metal nanoparticles on a silicon waveguide. A point dipole treatment is used, including the effects of the waveguide on both the angular emission spectrum and scattering cross section of the dipoles. The model agrees very well with our experimental results of greatly enhanced electroluminescence and photocurrent from silicon-on-insulator light-emitting diodes and also gives very good agreement with previously reported diffuse reflectance measurements. The results suggest that the main mechanism in the enhancement of diffuse reflectance in this system is a dramatic enhancement in the scattering cross section of waveguided light, rather than a waveguide-mediated dipole-dipole interaction. We also put lower bounds on the radiative efficiency of scattering by the nanoparticles.
\end{abstract} (C) 2006 American Institute of Physics. [DOI: 10.1063/1.2226334]

\section{INTRODUCTION}

There is currently a great deal of interest in surface plasmons on metal surfaces and metal nanoparticles, and the often surprising optical properties associated with them. To give just two examples, surface plasmons have been associated with the extraordinary optical transmittance of hole arrays in metal films, where transmittances greater than unity (normalized to the area of the holes) have been observed; surface plasmon effects are also largely responsible for the factors of up to $10^{14}$ enhancement that have been observed using surface-enhanced Raman spectroscopy. ${ }^{2}$ A wide range of technological applications for surface plasmons are emerging. For example, surface plasmons provide a way to confine and transport light at subwavelength scales, which is of great interest in integrated optics. ${ }^{3}$ Surface plasmon effects also offer a range of possibilities for optoelectronics and photovoltaics, including improving selective emitters for thermophotovoltaics, and enhancing nonlinear processes for upconversion of low energy photons, ${ }^{4}$ along with increasing the emission of light-emitting diodes. ${ }^{5,6}$ In this paper we investigate promising near-term applications for surface plasmons in photovoltaics and optoelectronics: absorption/ emission enhancement due to dipole excitation of metal nanoparticles on a silicon waveguide.

Low absorptance at wavelengths in the region of the band edge is a significant issue for silicon solar cells and photodetectors, due to the indirect band gap of silicon. This is becoming more important as the thickness of silicon is reduced, to reduce the cost in the case of solar cells and to decrease the response time in the case of photodetectors. One way to increase the absorptance for solar cells, which have total thickness at least $1 \mu \mathrm{m}$, is to texture the surface of the silicon. However, this also increases the surface area, and hence increases the effect of surface recombination, which for high quality silicon is the limiting factor on the open-

${ }^{a)}$ Electronic mail: k.catchpole@unsw.edu.au circuit voltage for thin solar cells. Plasma etch techniques, which are generally required to etch submicron sized features, may also damage the silicon, further reducing cell efficiency. For photodetectors which may have a total silicon thickness of only a few hundreds of nanometers, texturing the silicon surface is clearly not an option, since feature sizes need to be at least $200-300 \mathrm{~nm}$ to diffract beyond the zeroth order.

Recently we have reported a dramatic enhancement in electroluminescence from silicon-on-insulator light-emitting diodes (SOI LEDs) by a factor of 8 times near the band edge at $900 \mathrm{~nm} .^{5}$ This built on earlier work of Stuart and Hall, who achieved an impressive increase in photogenerated current of 18 times at $800 \mathrm{~nm}$ in an SOI photodetector. ${ }^{7}$ Stuart and Hall also reported peaks in the diffuse reflectance spectrum, which they attributed to enhanced dipole-dipole interaction via waveguide modes. ${ }^{8}$ The diffuse reflectance spectrum has been modeled by Soller, using a convolution of the single particle Green's dyadic with a correlation function for the average properties of the particle distribution, ${ }^{9}$ and by Johannson, using a lattice-gas model with averaging over different realizations of the disorder using a coherent potential approximation. ${ }^{10}$ However, there has been no model proposed to date to predict the absorptance enhancement in the silicon.

In this paper we model the metal nanoparticles as noninteracting, ideal dipoles on the surface of the silicon waveguide. With this simplified model we are nevertheless able to achieve good agreement with many of the features of the absorptance enhancement and diffuse reflectance spectra and develop insight into the most important mechanisms in this system. By taking into account the angular dependence of both the emission spectrum and the scattering cross section we develop a consistent point dipole view of the effect on absorptance and scattering. We also consider how finite sized particles would tend to alter these results. 


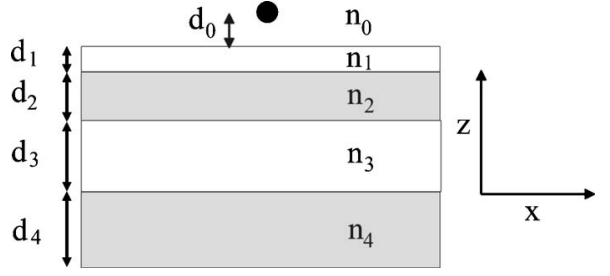

FIG. 1. The system considered in this paper. A dipole placed a distance $d_{0}$ from a waveguide.

\section{METHOD}

There are two major components to the model. The first is the calculation of the angular dependence of the scattered light emerging from the dipole and the angular dependence of the scattering cross section for incoming light. The second is the calculation of the light absorbed in the waveguide and the light coupled out of the waveguide. These are described in detail below.

\section{A. Angular dependence of scattering cross section and light emission for a dipole near a waveguide}

The calculation of the angular dependence of emission for a fixed amplitude dipole near an interface or waveguide is important in the study of fluorescence, and there have been many papers devoted to it. ${ }^{11-13}$ However, it appears to have been recognized only recently that for a dipole that scatters light rather than fluoresces, the presence of nearby interfaces can also strongly affect the scattering cross section, and indeed result in an angularly dependent scattering cross section. That this follows from the model of a dipole near an interface has been shown by Mertz. ${ }^{14}$

The results of Mertz's theory are summarized below. We consider the system shown in Fig. 1. A point dipole sits in a medium of refractive index $n_{0}$, a distance $d_{0}$ above a waveguide structure. For definiteness we show a four layer waveguide such as that in a SOI structure, defined by refractive indices $n_{1}, n_{2}, n_{3}$, and $n_{4}$, and distances $d_{0}, d_{1}, d_{2}$, and $d_{3}$, as shown in the figure. (In our experimental system the metal nanoparticles sit directly on the SOI structure and we take $d_{0}$ to be zero). by

The scattering cross section of a point dipole is defined

$$
\sigma=S_{\text {out }} / I_{\text {in }},
$$

where $S_{\text {out }}$ is the total scattered power and $I_{\text {in }}$ is the intensity of the input field, i.e.,

$$
I_{\text {in }}=\frac{1}{2} \varepsilon_{0} c n_{\text {in }}\left|E_{\text {in }}\right|^{2} .
$$

Here $\left|E_{\text {in }}\right|$ is the amplitude of the input field and the input field is incident from material with refractive index $n_{\text {in }}$. This is relevant for a particle on a waveguide because the particle may be excited by radiation either external or internal to the waveguide.

The total scattered power is equal to the work exerted by the dipole,

$$
S_{\text {out }}=\frac{\omega}{2} \operatorname{Im}\left(p^{*} E_{d}\right),
$$

where $\omega$ is the frequency of the incident light, $p$ is the particle polarization, and $E_{d}$ is the driving field. The waveguide can affect the scattering cross section by changing the dipole polarization or by changing the local driving field. The resulting expressions for the total scattered power for a dipole on a waveguide are

$$
\begin{aligned}
& S_{\text {out }}^{s}=\frac{\omega \varepsilon_{0}}{2} \frac{n_{\text {in }}}{n_{0}} \operatorname{Im}\left(\alpha_{h}\right) L_{h}^{s}\left(\theta_{\text {in }}\right)\left|E_{\text {in }}^{s}\right|^{2}, \\
& S_{\text {out }}^{p}=\frac{\omega \varepsilon_{0}}{2} \frac{n_{\text {in }}}{n_{0}}\left[\operatorname{Im}\left(\alpha_{h}\right) L_{h}^{p}\left(\theta_{\text {in }}\right)+\operatorname{Im}\left(\alpha_{v}\right) L_{v}^{p}\left(\theta_{\text {in }}\right)\right]\left|E_{\text {in }}^{p}\right|^{2} .
\end{aligned}
$$

Here $\alpha_{h}$ and $\alpha_{v}$ are the polarizabilities of the horizontal and vertical electric dipoles, respectively, in the presence of the waveguide. As $d_{0} \rightarrow \infty, \alpha_{h}, \alpha_{v} \rightarrow \alpha_{0}$, the polarizability of the dipole in free space. We also have

$$
\begin{aligned}
& L_{h}^{s}\left(\theta_{\text {in }}\right)=\frac{n_{0}}{n_{\text {in }}}\left|K_{h}^{s}\left(\theta_{\text {in }}\right)\right|^{2}, \\
& L_{h}^{s}\left(\theta_{\text {out }}\right)=\frac{n_{\text {out }}}{n_{0}}\left|K_{h}^{s}\left(\theta_{\text {out }}\right)\right|^{2},
\end{aligned}
$$

with corresponding definitions for $L_{h}^{p}$ and $L_{v}^{p}$. The expressions for $K$ are

$$
\begin{aligned}
& K_{h}^{p}\left(\theta_{\text {in }}\right)=\cos \theta_{0}\left[-\exp \left(-i k_{0 z} d_{0}\right)+r_{p} \exp \left(i k_{0 z} d_{0}\right)\right], \\
& K_{h}^{s}\left(\theta_{\text {in }}\right)=\exp \left(-i k_{0 z} d_{0}\right)+r_{s} \exp \left(i k_{0 z} d_{0}\right), \\
& K_{v}^{p}\left(\theta_{\text {in }}\right)=\sin \theta_{0}\left[-\exp \left(-i k_{0 z} d_{0}\right)-r_{p} \exp \left(i k_{0 z} d_{0}\right)\right],
\end{aligned}
$$

for externally incident light, and

$$
\begin{aligned}
& K_{h}^{p}\left(\theta_{\text {in }}\right)=-t_{p} \cos \theta_{0} \exp \left(i k_{0 z} d_{0}\right), \\
& K_{h}^{s}\left(\theta_{\text {in }}\right)=t_{s} \exp \left(i k_{0 z} d_{0}\right), \\
& K_{v}^{p}\left(\theta_{\text {in }}\right)=-t_{p} \cos \theta_{0} \exp \left(i k_{0 z} d_{0}\right),
\end{aligned}
$$

for internally incident light, where $k_{0 z}=2 \pi n_{0} \cos \left(\theta_{0}\right) / \lambda$ is the component of the wave vector perpendicular to the surface in region 0 . Here $r_{s}, r_{p}, t_{s}$, and $t_{p}$ are the Fresnel coefficients of the waveguide structure. $L$ represents the contribution of the local driving field to the scattering cross section of the dipole in the presence of the waveguide. Thus there are contributions to the scattering cross section from the waveguide via a change in the local field (through $L$ ) and via a change in the effective polarizability of the particle, through $\operatorname{Im}(\alpha)$. Note that $r_{s}$ and $r_{p}$ are the reflection coefficients for light incident from layer 0 , while $t_{s}$ and $t_{p}$ are the transmission coefficients for light incident from the bottom-most layer, in the present case layer 4. Also note that the angles of incidence may be imaginary corresponding to excitation of the dipole by evanescent waves.

The angular dependence of the scattering cross section described by Eq. (4) is a direct consequence of the Lorentz reciprocity theorem. For the case of a dipole near a wave- 
guide, this means that for the directions associated with the waveguide modes there is a strong coupling between the dipole and the waveguide. Most of the radiation emitted by a dipole is emitted in these directions, and incoming radiation from these directions is strongly scattered by the dipole.

The total emitted power is given by

$$
\begin{aligned}
& N_{h}=\frac{3}{8} \int_{0}^{\pi}\left[L_{h}^{s}(\theta)+L_{h}^{p}(\theta)\right] \sin \theta d \theta, \\
& N_{v}=\frac{3}{4} \int_{0}^{\pi} L_{v}^{p}(\theta) \sin \theta d \theta,
\end{aligned}
$$

where $N$ represents the total output power for a fixed amplitude oscillator (as opposed to an oscillator driven by a fixed amplitude incident field). The integrands are the power emitted into a solid angle characterized by $d \theta$, where $\theta$ is the angle from the normal. We can use Eqs. (8) to calculate the angular spectrum of the light emitted by a dipole. If we define the in-plane, normalized wave vector $u$ by $u=k_{0 x} / k_{0}$ $\left(=n_{2} \sin \theta_{2}\right)$, then for $u<1$ the emission is into the escape cone, for $1<u<n_{3}$ emission is into leaky modes, and for $u>n_{3}$ emission is into the guided modes of the waveguide. By selective integration of Eqs. (8) it is therefore possible to calculate the fraction of the radiation emitted by the dipole into the escape cone, into leaky modes and into guided modes.

The total emitted power also modifies the decay rate of the dipole and the Mertz model can be used to determine the effect of a waveguide on the polarizability of a particle via the change in decay rate. (There is also a similar derivation in Wasey and Barnes, ${ }^{15}$ where there is a detailed discussion of the effects of interfaces on dipole decay rates.) However, for the waveguides studied in this paper the effect of the waveguide on the polarizability is small and has no consequences for the conclusions presented here. Since there is a large effect of the local field on the calculated scattering cross sections and considerable uncertainty in the experimental scattering cross sections, we neglect the effect of the waveguide on the particle polarizability.

For the initial scattering cross-section spectrum before modification by the waveguide we use experimentally determined transmittance spectra for islands on glass. This is because simple models of the extinction of islands on glass only qualitatively reproduce the broadening seen experimentally. ${ }^{16}$ In a full model of the transmittance of islands on glass it would be necessary to include retardation effects for a single particle, distributions of particle shapes, ${ }^{17}$ and the effects of interaction between the particles in a disordered arrangement. ${ }^{18}$

While the method of Mertz provides a simple and transparent explanation for the change in angular spectrum and scattering cross section for a dipole near a waveguide, the effect of absorption in the silicon on the angular spectrum is more easily dealt with using the source term method of Benisty et al. ${ }^{19}$ We therefore use the source term method in calculating the angular emission spectra; this is described in more detail below.

\section{B. Calculation of absorption and outcoupling of scattered light}

The model used for the calculation of absorption and outcoupling is based on the transfer matrix method for thin film optics. ${ }^{20}$ It is a semicoherent model with a dipole model used to determine the angular spectrum of diffuse radiation. The flux incident on the first rough surface in the model is calculated using the transfer matrix technique. This flux is then divided into a coherent, nonscattered component and a diffuse component. The diffuse component is calculated according to elastic scattering for an ideal dipole and is assumed to be an incoherent process. The assumption of incoherence is expected to be a good approximation due to the disordered arrangement of the nanoparticles, and in any case can be tested by comparison with the experimental behavior of the system. Combined with the dipole angular emission spectrum this essentially means that the dipole interacts coherently with itself, but incoherently with other dipoles. After the flux at the rough interface has been divided into coherent and diffuse components, the fields due to the fluxes are calculated and propagated through the structure coherently by the transfer matrix technique, thus taking interference at all other interfaces through the structure into account. In this way the field, and hence the flux incident on the rough surface for the second scattering process, is calculated. This method is due to Leblanc et $a .^{21}$ and is described in more detail in that paper. These authors also introduced an elegant matrix method for calculating the effect of subsequent scattering processes from the results of the first scattering process, thus making the whole computation rather quick.

Leblanc et al.used Lambertian-type angular distributions for the diffuse flux in their model, with the diffuse reflectance and transmittance proportional to $\cos (\theta)$. This may be appropriate for textured $a$-Si solar cells with thicknesses of at least $1 \mu \mathrm{m}$, but it is not appropriate for Si devices of the order of $100 \mathrm{~nm}$ thick, that have a very limited number of guided modes available in which light can propagate. To take account of the angular spectrum of emission of the dipoles we used the method of Benisty et al., ${ }^{19}$ which is an extension of the transfer matrix method that treats the dipoles as source terms. It is equivalent to the method of Mertz for calculating the angular emission method for a fixed amplitude dipole in a weakly absorbing waveguide. The advantage of the source term method is that it fits very naturally with the flux propagation calculation that also uses the transfer matrix method. It is also more suitable than the method of Mertz at short wavelengths, where the waveguide is quite strongly absorbing. The calculations for the angular distribution of the emission of the dipoles were checked against the results of Benisty et al. ${ }^{19}$ and Lukosz. ${ }^{12}$ The source term method is for a fixed amplitude dipole. The results for the flux emitted in a particular direction were therefore normalized by the total emitted flux. For the scattering cross sections, Mertz's expressions were used as described in the next section.

The equation for the absorptance due to diffused light $A_{\text {tot }}$ from Leblanc et al. in the case where the front surface of the device is the scattering surface is 


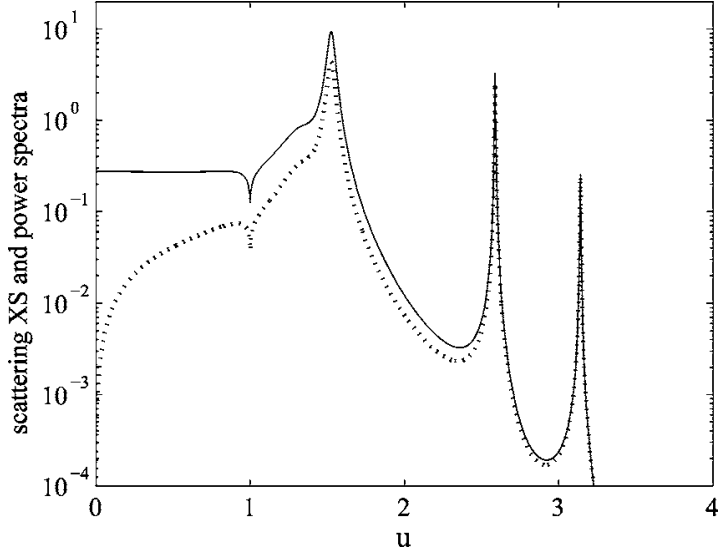

FIG. 2. Scattering cross section (solid line) and angular power spectrum (dotted line) for internally incident light in the region of the major waveguided mode for the $95 \mathrm{~nm}$ SOI structure at $900 \mathrm{~nm}$. It can be seen that the scattering cross section is high for the mode into which most of the light is scattered $(u \sim 1.5)$, whereas for the other modes at $u \sim 2.5$ and $\sim 3.2$ the scattering cross section is much lower. For waveguided light incident on the dipole with the angular spectrum given above, $70 \%$ has a scattering cross section above 1.

$$
A_{\text {tot }}=[A][I-F]^{-1} \Sigma_{0+},
$$

where $\Sigma_{0+}$ is the incident flux from layer 0 and $A$ is a matrix that describes the absorptance that occurs between subsequent scattering processes. $F$ is a matrix describing the flux remaining within the device after each scattering process and $[I-F]^{-1}$ is the geometric sum of the flux remaining in the device, where $I$ is the identity matrix.

We can obtain a similar equation for the diffuse reflectance $R_{d, \text { tot }}$.

$$
R_{d, \text { tot }}=\frac{\Sigma_{1-}}{\Sigma_{2-}}[I-F]^{-1} \Sigma_{0+} .
$$

Here $\Sigma_{1-} / \Sigma_{2-}$ is the fraction of flux that exits the device into air, compared to the flux that is incident on the scattering surface. The effect of an angularly depending scattering cross section on the above equations is made explicit in the following sections.

\section{RESULTS}

In this paper we give modeled results for two particular SOI structures. The first is the structure used for our initial experiments with SOI light-emitting diodes. This had a $408 \mathrm{~nm}$ buried oxide with a $95 \mathrm{~nm}$ active Si layer and $56 \mathrm{~nm}$ top oxide. The second is the structure used by Stuart and Hall for their SOI photodetector, with $205 \mathrm{~nm}$ buried oxide, $160 \mathrm{~nm}$ active Si layer, and $30 \mathrm{~nm} \mathrm{LiF}$ spacer layer. ${ }^{7}$

\section{A. Effect of the angular dependence of the emission on the scattering cross section}

Figure 2 shows the power spectrum for emission and the normalized scattering cross section versus the normalized inplane wave vector $u$ for the $95 \mathrm{~nm}$ SOI structure at $900 \mathrm{~nm}$ for light incident from the Si side of the device. We can see that the emitted power is strongly concentrated in the mode at $u \sim 1.5$, with minor additional modes at $u \sim 2.5$ and 3.2. The scattering cross section (which has been normalized by

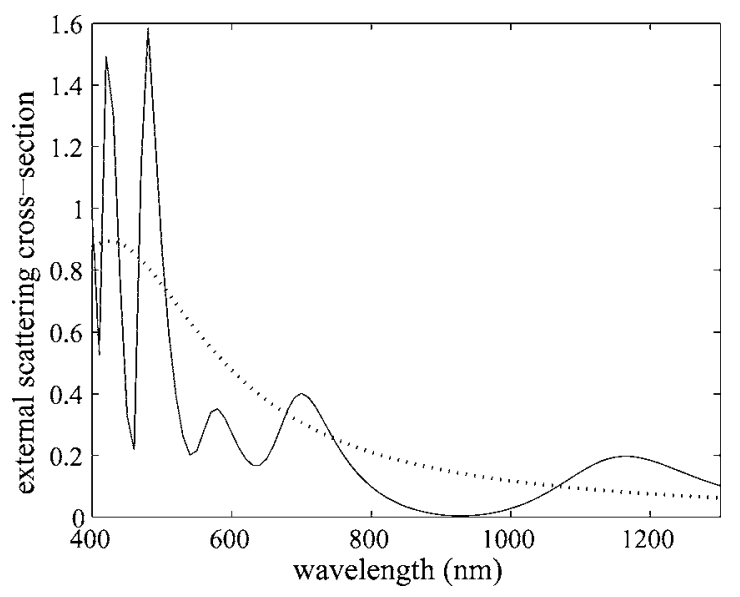

FIG. 3. Scattering cross sections for external, normally incident light for the $160 \mathrm{~nm}$ SOI structure with (solid line) and without (dotted line) modification of the cross section by the waveguide.

the average area per particle, i.e., the inverse particle density) has a similar structure and is highest in the region in which most of the power is emitted. Thus for the majority of the light incident on the dipole from the waveguide modes the normalized scattering cross section is above 1 and, in fact, reaches up to around 9. (A normalized scattering cross section above 1 means that the particle can scatter light that is incident on an area larger than the average area per particle, i.e., potentially all of the incident light could be scattered even if the density of nanoparticles on the surface was lower.) The strong coupling that results in most of the power emitted from a dipole going into a few waveguide modes also results in a dramatic enhancement of the scattering cross section for light incident on the dipole from those waveguide modes. Since the light from the dipole is radiated into a narrow range of values which have very high scattering cross sections, and since repeated scattering events tend to further concentrate this light into this narrow range of angles, to a very good approximation we can simply say that all waveguided light incident on the dipole is scattered.

Figure 3 shows the scattering cross-section spectrum for externally incident light for islands on glass. The corresponding spectrum as modulated by the waveguide according to the Mertz model is also shown, for the $160 \mathrm{~nm}$ SOI structure. It can be seen that the point dipole model affects the scattering cross section for externally incident light, as well as for internally incident light. The cross section peaks at the maxima of $L_{s}\left(\theta_{\mathrm{in}}=0\right)$, i.e., where $\left|1+r_{s}\right|^{2}$ reaches its maximum values. Thus the scattering cross section is enhanced when the local field due to the waveguide is high.

Taking into account the modification of the scattering cross sections with incident angle, the final equations for the absorptance due to diffused light $A_{\text {tot }}$, and diffuse reflectance $R_{d, \text { tot }}$ are

$$
A_{\text {tot }}=\sigma\left(\theta_{\text {in }}, \lambda\right)[A][I-F]^{-1} \Sigma_{0+}
$$



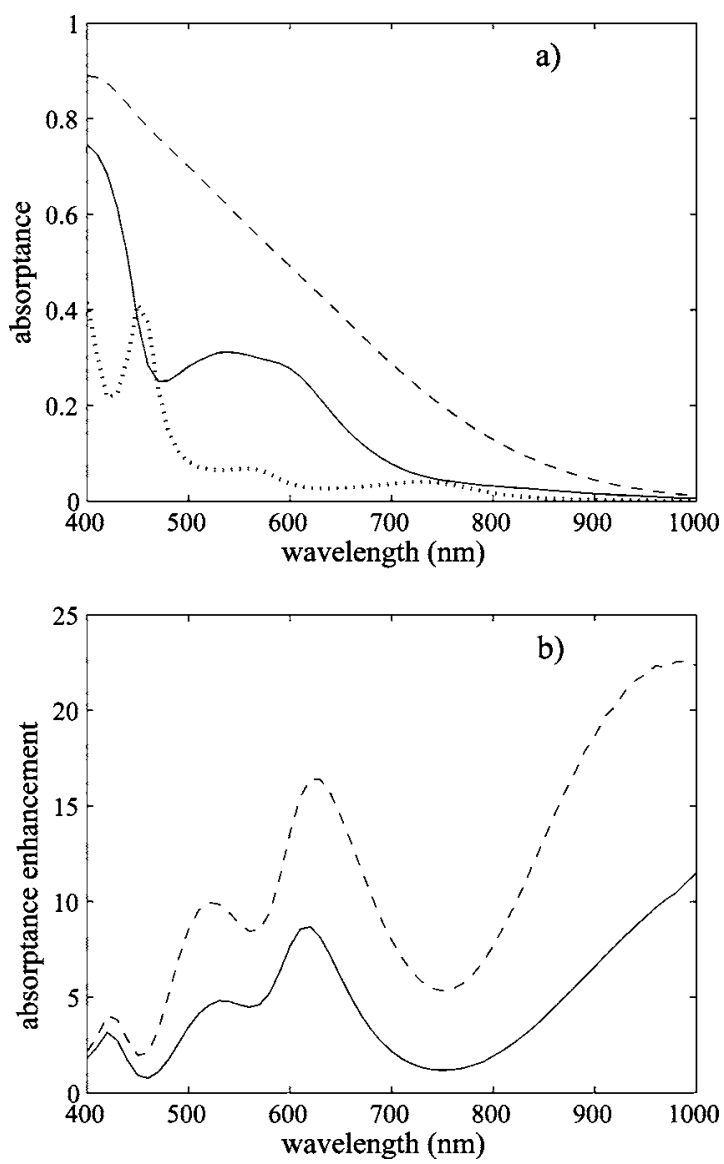

FIG. 4. (a) Absorptance for $95 \mathrm{~nm}$ SOI structure for the cases of a structure without nanoparticles (dotted line) assuming 100\% scattering with dipole angular spectrum (solid line) and assuming 100\% scattering with a Lambertian angular dependence (dashed line, for the purposes of comparison only since this is physically unrealistic for such a thin structure). (b) Absorptance enhancement for these cases.

$$
R_{d, \mathrm{tot}}=\sigma\left(\theta_{\mathrm{in}}, \lambda\right) \frac{\Sigma_{1+}}{\Sigma_{2-}}[I-F]^{-1} \Sigma_{0+},
$$

where $[A]$ and $[F]$ are calculated assuming $100 \%$ scattering of the waveguided incident light and $\sigma$ is the scattering cross section for externally incident light.

Both the model presented here and the waveguidemediated dipole-dipole interaction model ${ }^{8}$ take into account the variation with wavelength in the fraction of light emitted by the dipole into the waveguide and into air. The differences between the models are that in the model presented here the scattering cross section is angle as well as wavelength dependent, outcoupling effects are included, and there is no waveguide-mediated enhancement by nearby particles.

\section{B. Effect of the angular dependence of the emission on the absorptance}

Before describing the results for the absorption enhancement and diffuse reflectance calculated with Eqs. (11) and (12) we first look at the maximum potential absorption enhancement for a dipole on a waveguide. Figure 4 shows the results of the optical model for our $95 \mathrm{~nm}$ SOI structure assuming all incident light is scattered by the dipoles, showing the potential absorption enhancement predicted by the dipole angular spectrum compared with a Lambertian angular spectrum (although a Lambertian angular spectrum is not physically realistic for such a thin structure). It can be seen that the dipole angular spectrum results in a reduction in potential enhancement. This is due to the limited photonic density of states available in a waveguide structure. ${ }^{22}$

The results above are for the horizontal electric dipole (HED) as we have found that the experimental results are best predicted using only the HED. There are two likely reasons for this. Firstly, only the HED is initially excited by normally incident light. Subsequently, it is likely that effectively only the part of the metal nanoparticle very close to the interface is excited by the guided modes, i.e., the evanescent wave will "see" mostly the closest part of the nanoparticle. For the HED, the effective particle size will be approximately the diameter of the particle (for slightly flattened particles). However, for the vertical electric dipole (VED), the effective particle size will only be a small fraction of the height of the particle, leading to a much lower effective polarizability of the VED for guided mode excitation.

\section{Combined effects of the angular dependence of the scattering cross section and emission on the absorption and diffuse reflectance}

The combined effects of the modulation in scattering cross section and angular emission spectrum on the diffuse reflectance spectra for the $160 \mathrm{~nm}$ SOI structure are shown in Fig. 5. Figure 5(a) shows the case where the scattering cross section is unmodified by the waveguide; in Fig. 5(b) the dramatic effect of the modification of the scattering cross section for waveguided light is evident.

Considering first the case where the scattering cross section is unmodified by the waveguide, the diffuse reflectance after the first scattering event [dashed line in Fig. 5(a)] peaks at the points where the fraction of light emitted into the air reaches a maximum. By reciprocity these are also the wavelengths at which the field would reach a maximum due to constructive interference if the sample was illuminated over the whole hemisphere in air, i.e., where

$$
N_{h}^{\text {air }}=\frac{3}{8} \int_{0}^{\pi / 2}\left[L_{h}^{s}\left(\theta_{0}\right)+L_{h}^{p}\left(\theta_{0}\right)\right] \sin \theta_{0} d \theta_{0}
$$

reaches its maximum values, where $\theta_{0}$ is the angle in air. The high refractive index of the waveguide means that $L_{s}$ and $L_{p}$ are not strongly dependent on the angle in air, i.e., the wavelengths defined by Eq. (13) are also close to the wavelengths where $L_{s}\left(\theta_{\text {in }}=0\right)$ is at its maximum values. The diffuse reflectance including outcoupling effects [solid line in Fig. $5(\mathrm{a})]$ is redshifted compared to these values because of the decreasing absorption in the silicon at longer wavelengths. If the scattering cross section was unaffected by the waveguide, these two effects would completely describe the diffuse reflectance spectrum. The effects of outcoupling are relatively weak in this case because at the long wavelengths where outcoupling becomes significant, the scattering cross section is fairly small. The diffuse reflectance in the case where the scattering cross section is modified by the waveguide according to the point dipole model is qualitatively different, as can 

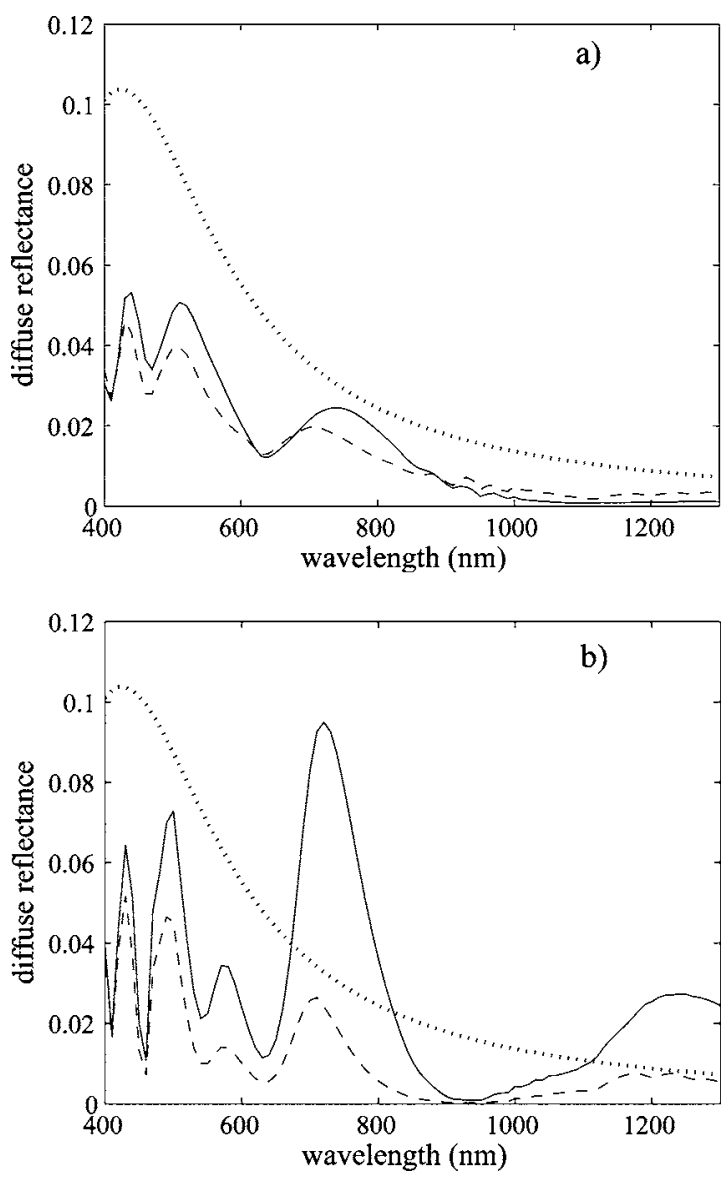

FIG. 5. Diffuse reflectance for the $160 \mathrm{~nm}$ thick Si layer SOI structure for the cases (a) with unmodified scattering cross sections and (b) with waveguide-modified scattering cross sections. The dashed line is the diffuse reflectance due to the first scattering event, the solid line is the full diffuse reflectance including outcoupling, and the dotted line is the diffuse reflectance for samples on glass, for the purpose of comparison.

be seen in Fig. 5(b). The peaks are narrower, due to the dependence of the external scattering cross section on $L_{s}\left(\theta_{\text {in }}=0\right)$. The long wavelength peaks are also much bigger, and the reason for this is the enhanced scattering of waveguided light. Comparison with the experimental data ${ }^{8}$ shows that the model of waveguide-modified scattering cross section described here provides a very good prediction of the diffuse reflectance for this system.

Although the modification of the scattering cross section for guided waves strongly affects the diffuse reflectance, it only has a relatively small effect on the absorptance, because most of the waveguided light that is scattered by the dipole is reemitted back into the waveguide. The effects of the modulation in scattering cross section and angular emission spectrum on the absorptance enhancement are compared with our experimental results ${ }^{23}$ for the $95 \mathrm{~nm}$ SOI structure in Fig. 6 . We can see that there is good agreement between the model and the experimental values in the positions, widths, and heights of the peaks.

The results for the absorptance enhancement for the $160 \mathrm{~nm}$ SOI structure are plotted in Fig. 7. For the $160 \mathrm{~nm}$ SOI structure the unmodified scattering cross-section model leads to increased absorptance at long wavelengths, which is also seen in the experimental data. This suggests that for
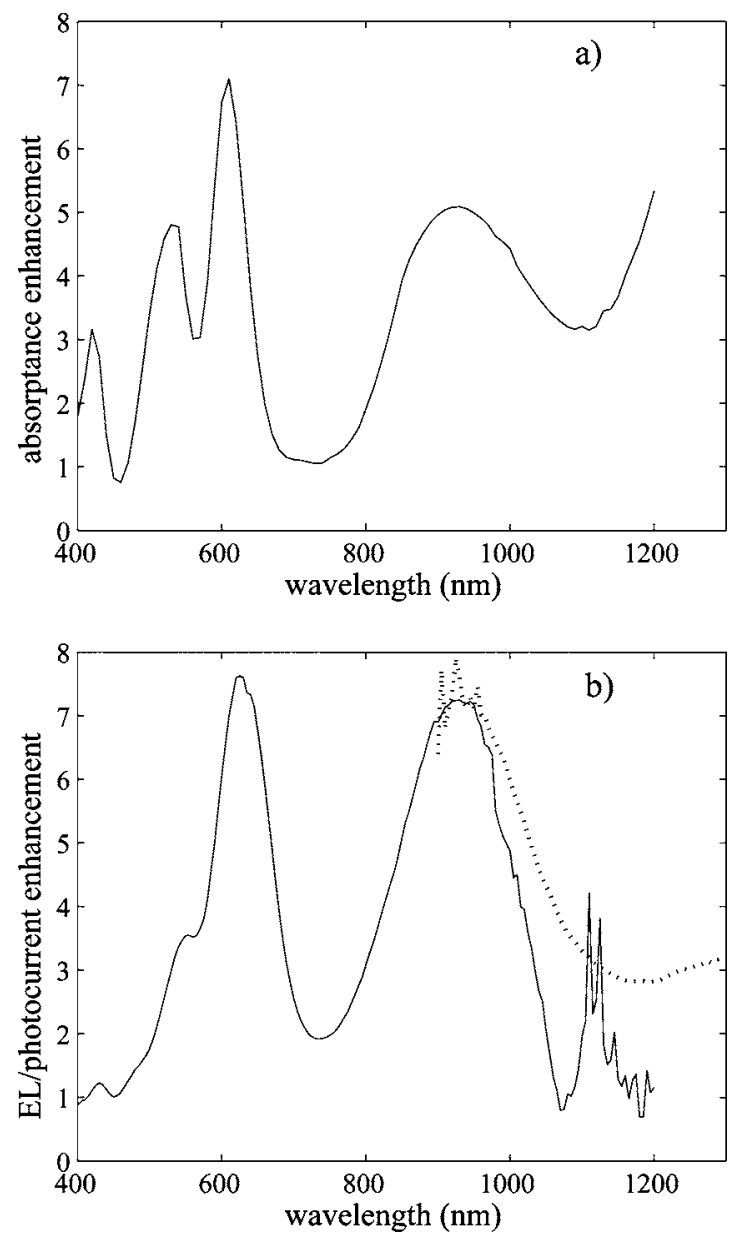

FIG. 6. (a) Modeled and (b) measured absorptance enhancements for a $95 \mathrm{~nm}$ thick Si layer SOI structure.

finite sized particles the modification of the external scattering cross-section may be decreased. This is in contrast to the internal scattering cross section, where the diffuse reflectance results indicate that the large enhancement of scattering cross section for waveguided light is still present for finite sized particles.

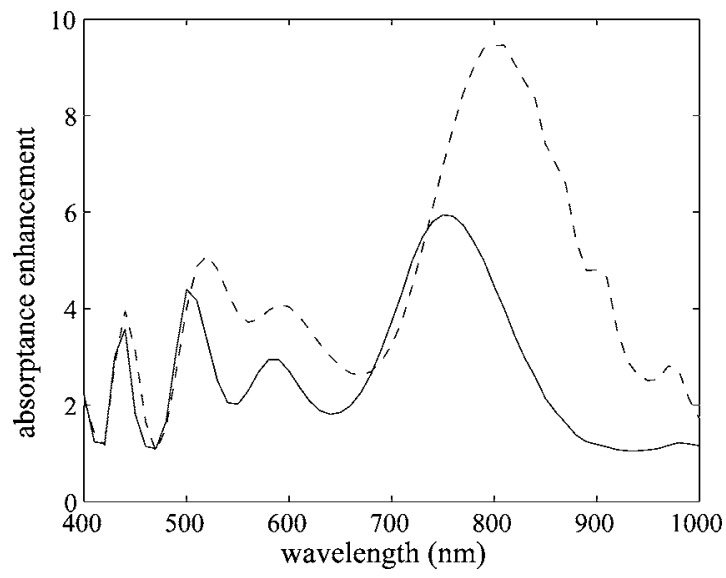

FIG. 7. Absorptance enhancement for a $160 \mathrm{~nm}$ thick Si layer SOI structure. The peaks are at $450,500-520,580$, and $750-800 \mathrm{~nm}$, which is in excellent agreement with the peaks seen at $450,525,600$, and $800 \mathrm{~nm}$ by Stuart and Hall (Ref. 7). The solid line is with waveguide-modified scattering cross sections, and the dashed line is with unmodified scattering cross sections. 

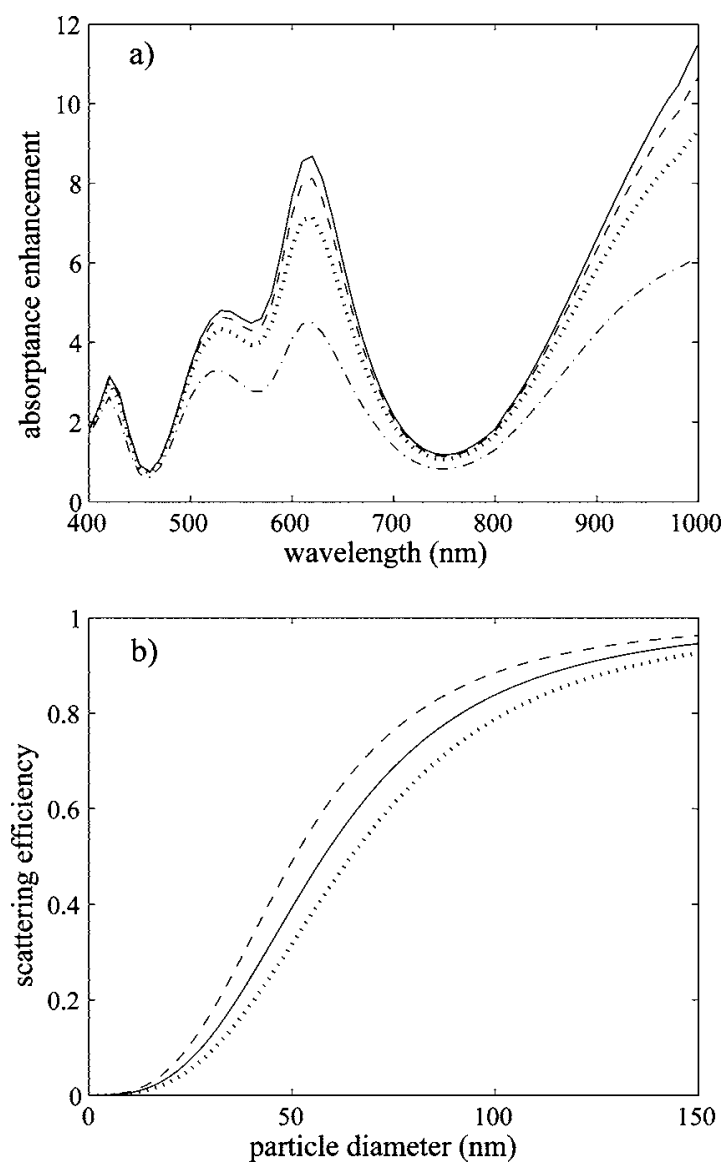

FIG. 8. (a) Effect of radiative efficiency on the absorption enhancement for the $95 \mathrm{~nm}$ structure, assuming 100\% scattering and either 100\% radiative efficiency (solid line), $150 \mathrm{~nm}$ diameter particles (dashed line), $100 \mathrm{~nm}$ particles (dotted line), or $50 \mathrm{~nm}$ particles (dash-dot line). (b) Calculated radiative efficiency vs particle diameter at wavelengths of $400 \mathrm{~nm}$ (dotted line), $600 \mathrm{~nm}$ (dashed line), and $1000 \mathrm{~nm}$ (solid line) for silver spheres.

\section{Effect of radiative efficiency of scattering}

We can put lower bounds on the radiative efficiency of silver nanoparticles by comparing experimental results with the absorption enhancement that is predicted by assuming $100 \%$ scattering of externally incident light. At each scattering event, only the flux equal to the incident flux multiplied by the radiative efficiency is reemitted, the rest is assumed to be lost as heat. Figure 8(a) below shows that the experimentally measured enhancement of a factor of 7.5 at $620 \mathrm{~nm}$ for the $95 \mathrm{~nm}$ SOI structure gives a lower bound for the radiative efficiency of $\sim 85 \%$. Because less than $100 \%$ of the incident light is actually scattered in the experiment at $620 \mathrm{~nm}$, the real radiative efficiency is likely to be significantly higher.

The radiative efficiency $Q_{\mathrm{rad}}=C_{\mathrm{sca}} /\left(C_{\mathrm{sca}}+C_{\mathrm{abs}}\right)$ for a small spherical particle in vacuum can be calculated from ${ }^{24}$

$$
C_{\text {abs }}=\frac{2 \pi}{\lambda} \operatorname{Im}[\alpha], \quad C_{\mathrm{sca}}=\frac{1}{6 \pi}\left(\frac{2 \pi}{\lambda}\right)^{4}|\alpha|^{2},
$$

where $\alpha=3 V(\varepsilon-1)(\varepsilon+2) . V$ is the particle volume and $\varepsilon$ is the dielectric function of the particle material. The radiative efficiency versus particle diameter at $620 \mathrm{~nm}$ for silver spheres is shown in Fig. 8(b). The value for the radiative efficiency predicted by Eqs. (14) at $620 \mathrm{~nm}$ is $94 \%$ for
$130 \mathrm{~nm}$ diameter particles, in agreement with the lower bound obtained from the absorptance enhancement measurements. We can expect that quadrupole contributions remain negligible for $130 \mathrm{~nm}$ diameter particles over most of the wavelength range considered since the ratio of the electric quadrupole to electric dipole scattering cross sections as calculated from the Mie theory ${ }^{25}\left[3 \operatorname{Im}\left({ }^{e} B_{2}\right) / \operatorname{Re}\left({ }_{e} B_{1}\right)\right]$ for silver sphere is 0.06 for a wavelength of $500 \mathrm{~nm}$ and decreases with wavelength; there may be a small quadrupole contribution around $400 \mathrm{~nm}$ where the ratio is 0.1 .

Note that the radiative efficiencies we calculate in Fig. 8(b) are much lower than those calculated by Stuart and Hall, ${ }^{7}$ this appears to be because their results were calculated versus particle radius rather than diameter as stated in their paper. This means that it is necessary to have a particle radius of at least $100 \mathrm{~nm}$ for high radiative efficiency. This would also explain the dramatic rise in absorption enhancement as the particle size is increased from 66 to $108 \mathrm{~nm}$ observed in that paper.

\section{DISCUSSION}

We have shown that for point dipoles the angular dependence of the dipole emission leads to a very high scattering cross section for waveguided light and that this leads to a dramatic enhancement in the diffuse reflectance at long wavelengths. For finite sized particles the interaction between the particle and the waveguide will be reduced, compared to the case of a point dipole. The consequences of this will be that there will be less modification by the waveguide of the external scattering cross section. For finite particles the fraction of light emitted into the waveguide is also reduced although it is still high. ${ }^{26}$ The internal scattering cross section will therefore also be reduced. However, because the waveguide results in such a large enhancement in the internal scattering cross section, we propose that even for finite size particles and at long wavelengths, essentially all of the light incident from waveguided modes is scattered. We can see from Fig. 2 that the scattering cross section for the guided mode could be reduced by a factor of 9 and still remain above 1 for some values of in-plane wave vector. We also note that the enhanced scattering for waveguided light seen in this system, which is due to multiple scattering between the particle and the interfaces of the waveguide, appears to be the same phenomenon that results in greatly enhanced scattering for single particles in waveguides in a wide variety of systems including electron scattering in a quantum wire, ${ }^{27}$ radiation pressure on particles, ${ }^{28}$ and the modification of the scattering cross-section for bubbles near an ocean surface. $^{29,30}$

Johansson has also suggested that the enhanced diffuse reflectance is a single particle effect and has pointed out that the externally incident light creates peaks in the local field in the presence of the waveguide, leading to an increase in the diffuse reflectance at these wavelengths. ${ }^{10}$ However, Johansson did not consider the role of the scattering cross section of the waveguided light. It can be seen from Fig. 3 that for externally incident light, the enhancement of the scattering cross section due to the waveguide at $710 \mathrm{~nm}$ is relatively 
small and that this does not account for the dramatic difference in diffuse reflectance between Figs. 5(a) and 5(b).

The variation of extinction data for island films means that it is not possible to make fully quantitative predictions of the absorptance and diffuse reflectance at this point, even with the use of experimental data as input to the model. While it is possible to achieve close repeatability in resonance spectra of island films, great care needs to be taken to do so. ${ }^{31}$ Therefore extinction spectra measured for islands on glass cannot be taken as providing quantitative information about islands on a waveguide. It has also been shown that the extinction of metal particles is very sensitive to the shape of the particle. ${ }^{32}$ The shape of the islands formed may vary with the substrate used even if the same spacer layer is used in each case since the substrate may affect the morphology or other properties of the thin spacer layer.

Within wavelength ranges where the number of waveguided modes does not change (e.g., $800-1050 \mathrm{~nm}$ for our $95 \mathrm{~nm}$ structure), the exact position of the peaks depends on the initial scattering cross section before modification by the waveguide and the radiative efficiency. Particles with a lower scattering cross section and lower radiative efficiency will tend to have absorptance and diffuse reflectance peaks blueshifted compared to larger particles with higher scattering cross sections and radiative efficiency. This is because the shorter, more strongly absorbed wavelengths contribute more in this case. We have seen this experimentally where the samples with the highest values of absorptance enhancement for the long wavelength peak also show the largest redshift. These samples must have more strongly scattering particles on the surface, perhaps because the particles are slightly larger.

\section{CONCLUSION}

We have developed an optical model for absorption enhancement and diffuse reflectance by metal nanoparticles on a silicon waveguide. A point dipole treatment is used, including the effects of the waveguide on both the angular spectrum and scattering cross-section of the dipoles. The model agrees very well with our experimental results of greatly enhanced electroluminescence and photocurrent from siliconon-insulator light-emitting diodes and also gives very good agreement with the diffuse reflectance measured by Stuart and Hall. Comparison of the model with our experimental results allows us to put a lower bound on the radiative scattering efficiency of our particles of $85 \%$ at $620 \mathrm{~nm}$. The good agreement between the model and the experiment suggests that the main mechanism in the enhancement of diffuse reflectance in this system is a single dipole-waveguide interac- tion, resulting in a dramatic enhancement in the scattering cross section of waveguided light, rather than a waveguidemediated dipole-dipole interaction.

\section{ACKNOWLEDGMENTS}

One of the authors (K.R.C.) acknowledges the support of an Australian Research Council fellowship. The authors acknowledge the support of the Centre of Excellence for Advanced Silicon Photovoltaics and Photonics, supported by the Australian Research Council.

${ }^{1}$ T. Ebbesen, H. J. Lezec, H. F. Ghaemi, H. F. Thio, and P. A. Wolff, Nature (London) 391, 667 (1998).

${ }^{2}$ S. Nie and R. Emory, Science 275, 1102 (1997).

${ }^{3}$ W. L. Barnes, A. Dereux, and T. W. Ebbesen, Nature (London) 424, 824 (2003).

${ }^{4}$ K. R. Catchpole, S. Pillai, and K. L. Lin, Proceedings of the World Conference on Photovoltaic Solar Energy Conversion, Osaka, p. 2714, 2003.

${ }^{5}$ S. Pillai, K. R. Catchpole, T. Trupke, G. Zhang, J. Zhao, and M. A. Green, Appl. Phys. Lett. 88, 161102 (2006).

${ }^{6}$ W. L. Barnes, J. Lightwave Technol. 17, 2170 (1999).

${ }^{7}$ H. R. Stuart and D. G. Hall, Appl. Phys. Lett. 73, 3815 (1998).

${ }^{8}$ H. R. Stuart and D. G. Hall, Phys. Rev. Lett. 80, 5663 (1998).

${ }^{9}$ B. Soller, Ph.D. thesis, University of Rochester, 2002.

${ }^{10}$ P. Johansson, Phys. Rev. B 64, 165405 (2001).

${ }^{11}$ G. W. Ford and W. H. Weber, Phys. Rep. 113, 195 (1984).

${ }^{12}$ W. Lukosz, J. Opt. Soc. Am. 69, 1495 (1979).

${ }^{13}$ W. L. Barnes, J. Mod. Opt. 45, 661 (1998).

${ }^{14}$ J. Mertz, J. Opt. Soc. Am. B 17, 1906 (2000).

${ }^{15}$ J. A. E. Wasey and W. L. Barnes, J. Mod. Opt. 47, 725 (2000).

${ }^{16}$ T. Gotz, W. Hoheisel, M. Vollmer, and F. Trager, Z. Phys. D: At., Mol. Clusters 33, 133 (1995).

${ }^{17}$ P. Royer, J. L. Bijeon, J. P. Goudonnet, T. Inagaki, and E. T. Arakawa, Surf. Sci. 217, 384 (1989).

${ }^{18}$ R. G. Barrera, M. d. Castillo-Mussot, G. Monsivais, P. Villasenor, and W. L. Mochan, Phys. Rev. B 43, 13819 (1991).

${ }^{19}$ H. Benisty, R. Stanley, and M. Mayer, J. Opt. Soc. Am. A 15, 1192 (1998).

${ }^{20} \mathrm{P}$. Yeh, Optical Waves in Layered Media, edited by B. E. Saleh (Wiley-Interscience, New York, 1998).

${ }^{21}$ F. Leblanc, J. Perrin, and J. Schmitt, J. Appl. Phys. 75, 1074 (1994).

${ }^{22}$ H. R. Stuart and D. G. Hall, J. Opt. Soc. Am. A 14, 3001 (1997).

${ }^{23}$ S. Pillai, K. R. Catchpole, T. Trupke, G. Zhang, and M. A. Green, Proceedings of the European Photovoltaic Solar Energy Conference, Barcelona, p. 369, Vol. 1, 2005

${ }^{24}$ C. F. Bohren and D. R. Huffman, Absorption and Scattering of Light by Small Particles (Wiley-Interscience, New York, 1983).

${ }^{25}$ M. Born and E. Wolf, Principles of Optics (Cambridge University Press, Cambridge, 2001).

${ }^{26}$ B. J. Soller and D. G. Hall, J. Opt. Soc. Am. B 19, 1195 (2002).

${ }^{27}$ C. Kunze and R. Lenk, Solid State Commun. 84, 457 (1992).

${ }^{28}$ R. Gomez-Medina, P. San Jose, A. Garcia-Martin, M. Lester, M. Nieto-Vesperinas, and J. J. Saenz, Phys. Rev. Lett. 86, 4275 (2001).

${ }^{29}$ Z. Ye and C. Feuillade, J. Acoust. Soc. Am. 102, 798 (1997).

${ }^{30}$ D. K. Dacol and D. G. Roy, Phys. Rev. E 72, 036609 (2005).

${ }^{31}$ R. Gupta, M. J. Dyer, and W. A. Weimer, J. Appl. Phys. 92, 5264 (2002).

${ }^{32}$ T. A. Germer, Opt. Lett. 27, 1159 (2002). 